\title{
Scar Treatment Using 585-nam Pulsed Dye Laser
}

Yong Hyun Kim

Baek-kyu Kim

Department of Plastic and Reconstructive Surgery, Seoul National University Bundang Hospital, Seoul National University College of Medicine, Seongnam, Korea
Received May 20, 2016

Revised June 9, 2016

Accepted June 9, 2016

\section{Correspondence}

Baek-kyu Kim

Department of Plastic and Reconstructive Surgery, Seoul National University Bundang Hospital, 82, Gumi-ro 173 Beon-gil, Bundang-gu, Seongnam 13620, Korea

Tel: +82-31-787-7223

Fax: +82-31-787-4055

E-mail: plasrecondgmail.com

(C) Korean Society for Laser Medicine and Surgery

(c) This is an open access article distributed under the terms of the Creative Commons Attribution NonCommercial License (http://creativecommons.org/ licenses/by-nc/4.0) which permits unrestricted noncommercial use, distribution, and reproduction in any medium, provided the original work is properly cited.
There has always been a great interest in scar treatment amongst doctors, and as a result, various treatment methods have been developed. However, the clinical effectiveness of these methods remains controversial. Currently, there are many studies being conducted to develop a scar treatment through selective photothermolysis using laser therapy, based on vascular proliferation playing a key role in the creation of scars. Previously, argon, neodymium-doped yttrium aluminium garnet, and Carbon dioxide lasers were used to treat scars; currently, $585-\mathrm{nm}$ pulsed dye laser (PDL) treatment is being widely considered. Patients prefer laser treatments over other treatment methods since laser treatments are simple and almost pain-free. However, the outcomes of laser treatments vary depending on the laser settings, scar characteristics, skin conditions, and time of treatment. Therefore, in order to enhance the application of PDL in scar treatment, the above factors along with the side effects of treatment should be investigated thoroughly.

\section{Key words}

Pulsed dye laser; Hypertrophic scar; Keloid 


\section{INTRODUCTION}

Hypertrophic scars and keloids are generally very difficult to treat. In the past, many treatment attempts such as keloid removal, skin transplant, steroid injections, and compression bandages have been made. However, these treatments not only have varying degrees of effectiveness that require difficult procedures but also have limited complete recoveries. The erythema of linear scars caused by surgeries takes several weeks to months to heal and flatten. Scars that do not go through the normal healing process cause erythema that can turn into hypertrophic scars or keloids depending on hyperpigmentation or hypopigmentation. Lasers have been used to treat diverse types of scars. Argon, neodymium-doped yttrium aluminium garnet ( $\mathrm{Nd}: \mathrm{YAG}$ ), and carbon dioxide $\left(\mathrm{CO}_{2}\right)$ lasers were first introduced and their long-term treatment efficacies have been reported. 'Recently, 585-nm pulsed dye laser (PDL) has been used in studies to determine its effects on clinical symptoms, skin texture, elasticity, and pruritus (i.e., itchiness) of post-surgical scars. The results of PDL treatments vary depending on a number of factors, including the laser setting, fluences, session, initial treatment time, combination treatment, and skin type. This study aims to provide optimal laser settings for scar improvement in PDL scar treatment based on the indications of the patient.

\section{LASERS FOR SCARS}

Until now, lasers have been used to treat several skin disorders. Since the 1980s, lasers have been used especially to treat hypertrophic scars and keloids. ${ }^{2}$ After discovering that $\mathrm{Nd}: Y A G$ can treat hypertrophic scars by causing a reduction in collagen production through the irradiation of fibroblasts, other types of lasers such as argon, $\mathrm{CO}_{2}$ laser, and the presently used PDL were studied for their application in scar treatment. Results of these studies revealed that PDL could improve scar texture and pliability as well as reduce redness, size, pruritus, and pain. $^{1-3}$

\section{PDL MECHANISM}

The exact mechanisms for laser-based scar treatment are not yet known. Based on the fact that vascular proliferation plays a key role in the creation of scars, most theories focus on selective photothermolysis. It has been suggested that the light energy of the laser is absorbed by hemoglobin, which produces heat and causes coagulation necrosis. The coagulation necrosis leads to tissue hypoxia, which causes neocollagenesis, heating of collagen fibers, dissociation of disulfide bonds, and both subsequent collagen fiber realignment and remodeling. ${ }^{1-3}$ Since PDL plays a key role in vascular proliferation in the early phase of scar formation, performing scar treatment at this stage interrupts the increased blood flow before it reaches the scar. This study showed that PDL histologically promotes an increase in the concentration of matrix metalloproteinase (MMP), an enzyme that breaks down collagen and decreases the accumulation of type 3 collagen. ${ }^{2,3}$

\section{EFFECTS OF PDL FOR SCARS}

Alster et al. ${ }^{4}$ reported that 16 patients ( 15 females and 1 male) with hypertrophic burn scars received PDL treatments, from whom 40 scar regions were selected and studied. All patients had previous experiences with $\mathrm{CO}_{2}$ laser resurfacing, chemical peels, intralesional corticosteroids, silicone gel sheeting (SGS) excision, and/or grafting and pressure/massage therapy. However, no visible results were achieved. For 585-nm PDL, 5.0 to 6.5 $\mathrm{J} / \mathrm{cm}^{2}$ (7-mm spot) and 4.5 to $5.0 \mathrm{~J} / \mathrm{cm}^{2}$ (10-mm spot) fluences were used for the PDL treatments. The patients received laser treatments over a span of 6-8 weeks and were followed up for six months after the treatment. Two physicians evaluated the improvements on a scale from 0 to 4 ( $0=$ no improvement, $1=$ minimal, $2=$ moderate, $3=$ vast improvement). The evaluation scores were 2.5, 2, and 2 for face, trunk, and extremities, respectively.

Alster et al..$^{5}$ presented 14 cases 11 females and 3 males) of erythematous and hypertrophic scars caused by trauma or surgery. The patients were treated with 585$\mathrm{nm}$ PDL and the patients' skin types were categorized into types I and II. Fluences of $6.5-6.75 \mathrm{~J} / \mathrm{cm}^{2}$ were used and the laser spots were placed adjacent to one another without overlapping. Each patient underwent one to two sessions of laser treatment depending on the individual response to treatment with six weeks in between each session. Six months after laser treatment, two independent adult observers evaluated the improvements. The results showed that one pulsed dye laser treatment improved the scar erythema and flattening of hypertrophic scar by $57 \%$, while two treatments improved these scar characteristics by $83 \%$.

\section{EFFECTS OF PDL WITH COMBINED TREATMENT}

Alster et al. ${ }^{6}$ reported the results of a study on 22 pa- 
tients with hypertrophic breast reduction scars. The patients had never received scar treatments before and the scars occurred less than six months prior to the study. The treatment was conducted by randomly treating the scars with $585 \mathrm{~nm}$ PDL alone or combined with triamcinolone acetonide (TAC) (10 to $20 \mathrm{mg}$ ). Fluences of 4.5$5.5 \mathrm{~J} / \mathrm{cm}^{2}$ and $10 \mathrm{~mm}$ spot were used for two treatments separated by six weeks. Afterwards, the clinical improvement, scar pliability, and symptomatic improvement were evaluated. The group that received PDL alone showed enhancements in clinical improvement, scar pliability, and symptomatic improvement while the PDL + TAC group showed greater scar improvement.

Wittenberg et al. ${ }^{7}$ reported the results of a study on 20 patients that compared sectional PDL treatment and SGS. Fluences of $6.5-8 \mathrm{~J} / \mathrm{cm}^{2}$ and $5 \mathrm{~mm}$ spot size were used for PDL treatment, which was conducted four times on each patient with eight weeks in between treatments. For at least 12 hours a day, SGS was applied over a span of 24 weeks. Scar improvements, as described previously to assess pain, burning, erythema, elasticity, and volume, were observed in both the PDL and SGS groups. However, no significant differences were observed. Since PDL and SGS both showed improvements of scars, it is expected that an even greater scar improvement will be observed when SGS is applied after PDL treatment. Therefore, this matter warrants conducting further studies.

Asilian et al. ${ }^{8}$ reported the results of a study on 69 patients who participated in randomized clinical trials. Not all patients had received any scar treatment within 6 months before the study and they were divided into 3 groups before scar evaluations. Group 1 only received TAC $(10 \mathrm{mg} / \mathrm{ml})$ treatment while group 2 received TAC $(0.1 \mathrm{ml}$ of $40 \mathrm{mg} / \mathrm{ml}$ ) and 5-FU (5-fluorouracil, $0.9 \mathrm{ml}$ of $50 \mathrm{mg} / \mathrm{ml})$ treatments and group 3 received TAC $(0.1 \mathrm{ml}$ of $40 \mathrm{mg} / \mathrm{ml}$ ), $5-\mathrm{FU} 0.9 \mathrm{ml}$ of $50 \mathrm{mg} / \mathrm{ml}$ ), and PDL (5-7 J/ $\mathrm{cm}^{2}$ ) treatments. All groups received treatments every week for a course of eight weeks. Treatments using PDL were conducted on group 3 in weeks 1, 4, and 8. The scar height, erythema, pliability, and pruritus improved in all groups. Improvements over 50\% were observed in $20 \%$ of the subjects in group 1, 55\% of the subjects in group 2 , and $75 \%$ of the subjects in group 3 . However, atrophy and telangiectasia were observed in $37 \%$ of the subjects from the group that was treated with TAC. Scar treatment was more effective when different types of treatments were used in combination compared with PDL treatment alone.

\section{EFFECTS OF PDL DEPENDING ON SKIN TYPE}

First introduced by Alster et al. in the 1980s, PDL has been studied exclusively on Westerners, whose skin types were predominantly I, II, and III. ${ }^{4}$ Previous studies have revealed the effectiveness of PDL on patients with light skin (e.g., Caucasian) of types I, II, and III. ${ }^{4-6}$ However, there is a need to look into studies that were conducted on Asian subjects with darker skin types.

Chan et al. ${ }^{9}$ reported the results of a study conducted on 29 Chinese subjects. The subjects were divided into the prevention group, consisting of patients who received scars within six months of entering the study, and the treatment group, consisting of 27 patients who received scars more than six months of starting the study. Fluences of 7-8 J/cm and $5 \mathrm{~mm}$ spot size were used for PDL treatment, which was conducted 3-6 times on each subject with eight weeks in between the sessions. Depending on the location of the scars, they were assigned to face, chest, leg, abdomen, back, chin, arm, neck, or hip categories. The results showed that the scar thickness was improved by $0.6 \mathrm{~mm}$ in the prevention group while the scar thickness was improved by $0.3 \mathrm{~mm}$ in the treatment group. In the prevention group, $54 \%$ of subjects expressed that their scars improved while $66 \%$ of subjects in the treatment group expressed the same opinion. The correlation between scar location and improvement was not observed in this study.

The results of this study demonstrated that scar treatments using PDL are also effective on Asian patients with darker skin types. In order to use PDL as a scar treatment method on Asian patients, its fluences should be set higher than when used on Westerners. When the patient's skin type is IV or V, such as in the case of Chinese patients, the concentration of melanin in the dermis is higher than in Westerners and higher fluences should be used for desirable clinical endpoints since the melanin competes with hemoglobin for the target chromophore. Therefore, $8 \mathrm{~J} / \mathrm{cm}^{2}$ fluence should be used for scar treatment but should be reduced if complications such as blistering occur. When $8 \mathrm{~J} / \mathrm{cm}^{2}$ fluence was used in Chinese patients, blistering was observed in only $6 \%$ of the patients. The reason why occurrences of complications are relatively lower in higher fluences is that the epidermal melanin content reduces the possibility of complications. However, when high fluences are used in darker skinned patients, epidermal melanin absorption is produced and can cause poorer results. This study also compared the results of PDL treatments on scars occurring within 6 months of treatment and scars occurring longer than 
6 months before the treatment. Scar improvement was greater when PDL treatment was conducted on fresh scars that occurred within 6 months.

Kono et al..$^{10}$ reported the results of a study on 19 patients who were treated with PDL. The treatment was conducted using $6 \mathrm{~J} / \mathrm{cm}^{2}$ fluence and $7 \mathrm{~mm}$ spot size with a gap of 4 to 8 weeks between the sessions. There were six patients whose skin type was III and seven whose skin type was IV. The results showed that there was a $58.9 \%$ improvement in scar height and $75.7 \%$ improvement in erythema. Patients with non-darker (e.g., light) skin types and patients who received multiple treatment sessions ( $>3$ ) showed greater scar improvements. This study showed that PDL treatment was relatively less effective in patients with darker skin types than in patients with light skin types, which was due to the competition between hemoglobin-specific laser and melanin. Therefore, patients with darker skin types require multiple treatments $(>3)$.

\section{EFFECTS OF PDL DEPENDING ON FLUENCES}

To date, most studies on PDL have reported its scartreating effects using 5-7 J/cm² fluences. ${ }^{4-10}$ However, there are studies that reported the positive effects of low fluences.

Manuskiatti et al. ${ }^{11}$ reported the results of a study on 10 patients with median sternotomy scars that occurred within 6 months of treatment. The patients (skin type I = $1,\|=5\| I=2,, \mid V=2$ ) had never received scar treatments previously. The scars were divided into four segments and were treated with PDL using $5 \mathrm{~mm}$ spot size and 3, 5, and $7 \mathrm{~J} / \mathrm{cm}^{2}$ fluences. Six treatments were conducted on each patient with a gap of four weeks in between the sessions. The different fluences did not show significant differences in terms of scar height, erythema, and pliability but a greater improvement was observed using $3 \mathrm{~J} / \mathrm{cm}^{2}$ fluence. In addition, erosions were observed using 5 and 7 $\mathrm{J} / \mathrm{cm}^{2}$ fluences, which were treated with simple ointment dressing within seven days.

This study showed that PDL treatment is effective using low $3 \mathrm{~J} / \mathrm{cm}^{2}$ fluence and that adverse effects such as skin erosion are observed at higher fluences.

Most studies reported that fluences of $5-7 \mathrm{~J} / \mathrm{cm}^{2}$ proved to be effective for scar treatment and that high fluences are effective in patients with darker skin types, like Asians. ${ }^{9}$ However, high fluences can cause skin complications such as erosion or blister formation and the physician should take this fact into account when performing treatment. This study, which was conducted on patients with relatively less pigmented, light skin types, showed that complications arise in high fluences while PDL was effective in scar treatment when low fluences were used.

\section{LIMITATIONS OF PDL AND ADVERSE EFFECTS}

Overall, PDL is effective in scar treatment and improves skin volume, texture, pliability erythema, pain, and pruritus. However, adverse effects were also observed in PDL treatments. The most common adverse effect was postoperative purpura and other effects such as hyperpigmentation or hypopigmentation can also be observed. ${ }^{1-3,11}$ This is closely related to thermal injury of the epidermis from melanin absorption and is more frequently observed in patients with darker skin types. ${ }^{1-3}$

\section{CONCLUSION}

The normal wound healing process is composed of an inflammatory response, angiogenesis, and activities of both nearby connective tissues and epidermal cells. Wounds that do not go through the normal wound healing process can cause the formation of erythematous, hypertrophic scars, or keloids. Recently, despite the rapid advances in wound healing processes and metabolism, it was observed that hypertrophic scars and keloids do not disappear easily. Many treatment methods such as surgical excision, SGS, and intralesional steroid injections are being applied in an attempt to heal scars. However, outcomes are difficult to predict using these treatment methods and are frequently associated with prolonged treatment periods as well as severe side effects. In addition, patient compliance has decreased due to the inconvenient need for anesthesia, pain during treatment, and difficulty of treatment.

The inconveniences above are the reasons why laser treatments are preferred over other scar treatment methods. Laser treatments do not require general anesthesia and do not produce excessive pain thereby enabling convenient treatments. Among different laser treatment methods, treatment using 585-nm PDL is being studied widely and its effectiveness is being proven.

Treatment with PDL is effective in terms of scar volume, texture, pliability, erythema, pain, and pruritus. To produce desirable results and maximize effectiveness using PDL, the appropriate laser setting, method, patient's skin type, and treatment period should be taken into account.

Most studies have reported that fluences of $5-7 \mathrm{~J} / \mathrm{cm}^{2}$ proved to be effective for scar treatment. ${ }^{4-10}$ However, the results vary depending on the patient's skin type. In 
patients with whitish skin types I and II, PDL is effective using $5-7 \mathrm{~J} / \mathrm{cm}^{2}$ fluences. However, high fluences can easily cause adverse effects such as blister formation and purpura. Therefore, some studies claim that low fluences such as $3 \mathrm{~J} / \mathrm{cm}^{2}$ fluence should be used for scar treatments using PDL.' In addition, since the laser-targeted hemoglobin and melanin compete with each other in patients with darker skin types IV or V, high fluences should be used and multiple sessions ( $>3$ ) are needed to produce optimal results. ${ }^{10}$

Although PDL treatment is effective by itself, when used in combination with TX (TAC, 5-FU), it can produce even better results In addition, PDL treatment is more effective on scars that occur within 6 months before the time of treatment than on scars that occur longer than 6 months before the treatment. ${ }^{6-9}$

Treatments with PDL can cause side effects such as postoperative purpura, hypopigmentation, or hyperpigmentation. Better results could be achieved when the physicians use appropriate laser settings based on the patient's skin type and scar conditions while fully understanding the mechanisms of PDL treatment. With further studies, PDL treatment will become an important addition to the scar treatment field.

\section{REFERENCES}

1. Bouzari N, Davis SC, Nouri K. Laser treatment of keloids and hypertrophic scars. Int J Dermatol 2007;46:80-8.

2. Parrett BM, Donelan MB. Pulsed dye laser in burn scars: current concepts and future directions. Burns 2010;36:443-9.

3. de las Alas JM, Siripunvarapon AH, Dofitas BL. Pulsed dye laser for the treatment of keloid and hypertrophic scars: a systematic review. Expert Rev Med Devices 2012;9:641-50.

4. Alster TS, Nanni CA. Pulsed dye laser treatment of hypertrophic burn scars. Plast Reconstr Surg 1998;102:2190-5.

5. Alster TS. Improvement of erythematous and hypertrophic scars by the $585-\mathrm{nm}$ flashlamp-pumped pulsed dye laser. Ann Plast Surg 1994;32:186-90.

6. Alster T. Laser scar revision: comparison study of $585-\mathrm{nm}$ pulsed dye laser with and without intralesional corticosteroids. Dermatol Surg 2003;29:25-9.

7. Wittenberg GP, Fabian BG, Bogomilsky JL, Schultz LR, Rudner EJ, Chaffins ML, et al. Prospective, single-blind, randomized, controlled study to assess the efficacy of the 585-nm flashlamp-pumped pulsed-dye laser and silicone gel sheeting in hypertrophic scar treatment. Arch Dermatol 1999;135:104955.

8. Asilian A, Darougheh A, Shariati F. New combination of triamcinolone, 5-Fluorouracil, and pulsed-dye laser for treatment of keloid and hypertrophic scars. Dermatol Surg 2006:32:907-15.

9. Chan HH, Wong DS, Ho WS, Lam LK, Wei W. The use of pulsed dye laser for the prevention and treatment of hypertrophic scars in chinese persons. Dermatol Surg 2004;30:987-94.

10. Kono T, Erçöçen AR, Nakazawa H, Honda T, Hayashi N, Nozaki M. The flashlamp-pumped pulsed dye laser ( $585 \mathrm{~nm}$ ) treatment of hypertrophic scars in Asians. Ann Plast Surg 2003;51:366-71.

11. Manuskiatti W, Fitzpatrick RE, Goldman MP. Energy density and numbers of treatment affect response of keloidal and hypertrophic sternotomy scars to the 585-nm flashlamppumped pulsed-dye laser. J Am Acad Dermatol 2001;45:55765. 